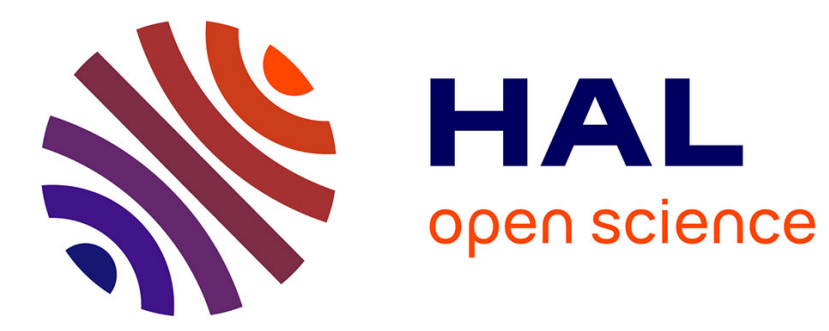

\title{
Doppler-free two-photon dispersion
}

\author{
G. Grynberg, M. Devaud, C. Flytzanis, B. Cagnac
}

\section{To cite this version:}

G. Grynberg, M. Devaud, C. Flytzanis, B. Cagnac. Doppler-free two-photon dispersion. Journal de Physique, 1980, 41 (9), pp.931-942. 10.1051/jphys:01980004109093100 . jpa-00208924

\section{HAL Id: jpa-00208924 https://hal.science/jpa-00208924}

Submitted on 1 Jan 1980

HAL is a multi-disciplinary open access archive for the deposit and dissemination of scientific research documents, whether they are published or not. The documents may come from teaching and research institutions in France or abroad, or from public or private research centers.
L'archive ouverte pluridisciplinaire HAL, est destinée au dépôt et à la diffusion de documents scientifiques de niveau recherche, publiés ou non, émanant des établissements d'enseignement et de recherche français ou étrangers, des laboratoires publics ou privés. 


\title{
Doppler-free two-photon dispersion
}

\author{
G. Grynberg, M. Devaud, C. Flytzanis \\ Laboratoire d'Optique Quantique du C.N.R.S., Ecole Polytechnique, 91128 Palaiseau, France \\ and B. Cagnac \\ Laboratoire de Spectroscopie Hertzienne de l'E.N.S., Université Pierre-et-Marie-Curie, 75230 Paris Cedex 05, France
}

(Reçu le 10 janvier 1980, accepté le 14 mai 1980)

\begin{abstract}
Résumé. - On présente dans cet article le calcul de la susceptibilité non-linéaire au voisinage d'une transition à deux photons sans élargissement Doppler. Dans le cas d'un système à deux niveaux, on donne une solution non perturbative qui permet de prévoir des effets nouveaux sur l'indice, effets liés au transfert de population entre niveaux fondamental et excité. Dans le cas d'un système plus complexe, on étudie à l'ordre le plus bas les effets d'anisotropie induits par un faisceau sur l'autre faisceau se propageant en sens inverse. On montre enfin que les conditions pour obtenir un régime bistable quand des atomes à l'intérieur d'une cavité Pérot-Fabry sont soumis à un champ dont la longueur d'onde est proche d'une résonance à deux photons, sont relativement faciles à réaliser.
\end{abstract}

\begin{abstract}
We present in this paper a calculation of the non-linear susceptibility near a Doppler-free two-photon transition. In the case of a two-level system, we find a non-perturbative solution. We deduce new effects on the non-linear refractive index which are related to the change of the linear susceptibility because of the transfer of atoms between the ground and excited levels. In the case of a more complex system, we study at the lowest order of perturbation the anisotropy induced by one beam on the other. At last, we show that it is easy to observe a bistable behaviour when atoms, inside a Pérot-Fabry cavity, interact with a beam whose wavelength is close to a two-photon resonance.
\end{abstract}

The problem of Doppler-free two-photon absorption has been considered in many papers [1]. On the other hand, only a very small number of papers [2] have discussed Doppler-free two-photon dispersion which is a subject closely related to the previous one. It certainly corresponds to the fact that Doppler-free two-photon absorption has been mostly used for spectroscopic purposes and in this case, it is generally easier and more precise [3] to detect the excited atoms by fluorescence or by ionization [4] rather than by the absorption itself. This explains why the evolution from saturated absorption spectroscopy to saturated dispersion which has been observed in a neighbour technique has little chance to occur with a great extension in the two-photon domain. Nevertheless, it seems interesting to calculate the formulae corresponding to two-photon dispersion, firstly because it may be possible that in some very particular cases the dispersion may be more accurate than the other detection mechanisms, secondly because the dependence of the index of refraction upon the intensity leads to new effects such as induced optical activity [2] and birefringence, bistability...
The paper is divided into four parts. In the first part, we perform a brief recall of the general formalism and we calculate the refractive index of a travelling wave when its wavelength is close to a two-photon resonance. In the second part, we consider the case where two waves of opposite directions interact in the medium and we calculate the modification of the refractive index of one wave due to the presence of the other. In the third part we describe two effects related with the interaction of the waves near a twophoton resonance : the optical activity [2] and the birefringence and we calculate the angular parameters in the general case of a $J_{\mathrm{g}} \rightarrow J_{\mathrm{e}}$ transition. In the last part, we consider the problem of optical bistability near a Doppler-free two-photon transition.

1. Refractive index of a travelling wave. 1.1 EfFective hamiltonian. - In order to describe the two-photon absorption, we need at least three levels : the ground and the excited states $g$ and $e$ and a relay level $j$ [3]. Nevertheless, it is possible in many cases to omit the level $\mathrm{j}$ by introducing the two-photon operator $[3,5]$ and setting up an effective hamilto- 
nian [6]. We now give a few details in order to clarify our notations.

In the following, the calculations dealing with the atom are always performed in the rest frame of the atom.

We consider an atom with two non-degenerate levels $|\mathrm{g}\rangle$ (ground state) and $|\mathrm{e}\rangle$ (excited state) of the same parity, and a certain number $r$ of relay levels $|j\rangle$ of the opposite parity. This atom interacts with an electric field whose value in its rest frame is equal to :

$$
\mathbf{E}(t)=\operatorname{Re} \varepsilon \varepsilon \mathrm{e}^{-i \tilde{\omega} t} .
$$

The interaction hamiltonian, at the electric dipole approximation, is equal to :

$$
V=-\mathbf{D} \cdot \mathbf{E}(t)
$$

where $\mathbf{D}$ is the electric dipole moment of the atom.

In order to solve the density matrix equation :

$$
i \hbar \frac{\mathrm{d}}{\mathrm{d} t} \rho=\left[H_{0}+V, \rho\right]-i \hbar \dot{\rho}_{\text {rel }}
$$

(where $H_{0}$ is the hamiltonian of the free atom and $\dot{\rho}_{\text {rel }}$ corresponds to the evolution due to relaxation), we assume that the following inequalities are satisfied :

$$
\left.\begin{array}{l}
\left|\omega_{\mathrm{jg}}-\tilde{\omega}\right| \\
\left|\omega_{\mathrm{je}}+\tilde{\omega}\right| \\
\left|\omega_{\mathrm{je}}-\tilde{\omega}\right|
\end{array}\right\} \gg\left\{\begin{array}{l}
\left|\omega_{\mathrm{eg}}-2 \tilde{\omega}\right| \\
\Omega
\end{array}\right.
$$

( $\hbar \omega_{\mathrm{jg}}$ is equal to the energy difference between the levels $\mathrm{j}$ and $\mathrm{g}$ and $\Omega^{-1}$ is the evolution time of the coherence $\tilde{\rho}_{\mathrm{ge}}$ in the interaction representation).

In that case we can integrate adiabatically the equations giving $i \hbar \frac{\mathrm{d}}{\mathrm{d} t} \rho_{\mathrm{je}}$ and $i \hbar \cdot \frac{\mathrm{d}}{\mathrm{d} t} \rho_{\mathrm{gj}}$ because the populations $\rho_{\mathrm{gg}}$ and $\rho_{\mathrm{ee}}$ and the coherence $\tilde{\rho}_{\mathrm{eg}}$ in the interaction representation vary very slowly with time (the other coherences $\rho_{\mathrm{jk}}$ where $\mathrm{j}$ and $\mathrm{k}$ are different from $\mathrm{g}$ and e can in most cases be neglected). Reporting the expressions obtained for $\rho_{\mathrm{gj}}$ and $\rho_{\mathrm{je}}$ in the equations giving $i \hbar \frac{\mathrm{d}}{\mathrm{d} t} \rho_{\mathrm{ee}}, i \hbar \frac{\mathrm{d}}{\mathrm{d} t} \rho_{\mathrm{gg}}$ and $i \hbar \frac{\mathrm{d}}{\mathrm{d} t} \rho_{\mathrm{ge}}$, we obtain, at the secular approximation, an equation for the density matrix restricted to subspace $\mathrm{g}$, e which is (in the Schrödinger representation) :

with

$$
\begin{gathered}
i \hbar \frac{\mathrm{d}}{\mathrm{d} t} \rho=\left[H_{\mathrm{eff}}, \rho\right]-i \hbar \dot{\rho}_{\text {rel }} \\
H_{\mathrm{eff}}=\left[\begin{array}{ll}
E_{\mathrm{e}}+\frac{|\varepsilon|^{2}}{4}\left\langle\mathrm{e}\left|Q_{\varepsilon \varepsilon^{*}}^{\prime}\right| \mathrm{e}\right\rangle & \frac{\varepsilon^{2}}{4}\left\langle\mathrm{e}\left|Q_{\varepsilon \varepsilon}\right| \mathrm{g}\right\rangle \mathrm{e}^{-2 i \omega t} \\
\frac{\varepsilon^{* 2}}{4}\left\langle\mathrm{~g}\left|Q_{\varepsilon^{*} \varepsilon^{*}}\right| \mathrm{e}\right\rangle \mathrm{e}^{2 i \omega t} & \frac{|\varepsilon|^{2}}{4}\left\langle\mathrm{~g}\left|Q_{\varepsilon^{*} \varepsilon}\right| \mathrm{g}\right\rangle
\end{array}\right]
\end{gathered}
$$

The energy of the ground state $E_{\mathrm{g}}$ has been taken equal to 0 and the $Q$ and $Q^{\prime}$ operators are those introduced in reference [6] :

$$
\begin{aligned}
& Q_{\varepsilon_{1} \varepsilon_{2}}=\mathbf{D} . \varepsilon_{1} \frac{1}{\hbar \tilde{\omega}-H_{0}} \mathbf{D} . \varepsilon_{2} \\
& Q_{\varepsilon_{1} \varepsilon_{2}}^{\prime}=\text { D. } \varepsilon_{1} \frac{1}{-\hbar \tilde{\omega}+E_{\mathrm{e}}-H_{0}} \text { D. } \varepsilon_{2}+\text { D. } \varepsilon_{2} \frac{1}{+\hbar \tilde{\omega}+E_{\mathrm{e}}-H_{0}} \text { D. } \varepsilon_{1} .
\end{aligned}
$$

(It can be noticed that the energy denominator $-\hbar \tilde{\omega}+E_{\mathrm{e}}-H_{0}$ is practically equal to $\hbar \tilde{\omega}-H_{0}$ because $\left|E_{\mathrm{e}}-2 \hbar \omega\right|$ is very small).

The hamiltonian is formally equivalent to that of a spin $1 / 2$ interacting with a static and a rotating magnetic field. The solution of such a system is well known, we find in the case of a weak relaxation a damped Rabi precession in the rotating frame. The Rabi pulsation $\Omega$ is equal to :

$$
\Omega=\sqrt{\left[\left(\omega_{\mathrm{eg}}-2 \tilde{\omega}\right)+\frac{s}{\hbar}\right]^{2}+\frac{1}{4}\left|\frac{\varepsilon^{2}\left\langle\mathrm{e}\left|Q_{\varepsilon \varepsilon}\right| \mathrm{g}\right\rangle}{\hbar}\right|^{2}}
$$

where $s$ is the light-shift of the g-e transition [6]

$$
s=\frac{|\varepsilon|^{2}}{4}\left[\left\langle\mathrm{e}\left|Q_{\varepsilon \varepsilon^{*}}\right| \mathrm{e}\right\rangle-\left\langle\mathrm{g}\left|Q_{\varepsilon^{*} \varepsilon}\right| \mathrm{g}\right\rangle\right] .
$$

In order to take into account the relaxation processes, we assume phenomenologically that :

$$
\begin{aligned}
& \left(\dot{\rho}_{\mathrm{ee}}\right)_{\mathrm{relax}}=-\Gamma_{\mathrm{e}} \rho_{\mathrm{ee}} \\
& \left(\dot{\rho}_{\mathrm{gg}}\right)_{\mathrm{relax}}=+\Gamma_{\mathrm{e}} \rho_{\mathrm{ee}} \\
& \left(\dot{\rho}_{\mathrm{eg}}\right)_{\mathrm{relax}}=-\Gamma_{\mathrm{eg}} \rho_{\mathrm{eg}} .
\end{aligned}
$$


The steady state solution is :

$$
\begin{aligned}
\rho_{\mathrm{ge}}=-\mathrm{e}^{2 i \omega t} \frac{\delta^{* 2}\left\langle\mathrm{~g}\left|Q_{\varepsilon^{*} \varepsilon^{*}}\right| \mathrm{e}\right\rangle}{4} \times \\
\times \frac{\delta \tilde{E}-i \hbar \Gamma_{\mathrm{eg}}}{\delta \tilde{E}^{2}+\hbar^{2} \Gamma_{\mathrm{eg}}^{2}\left(1+a^{2}\right)}
\end{aligned}
$$

$\rho_{\mathrm{eg}}=\rho_{\mathrm{ge}}^{*}$

$\rho_{\mathrm{ee}}=\frac{1}{2} \frac{a^{2} \hbar^{2} \Gamma_{\mathrm{eg}}^{2}}{\delta \tilde{E}^{2}+\hbar^{2} \Gamma_{\mathrm{eg}}^{2}\left(1+a^{2}\right)}$

$\rho_{\mathrm{gg}}=1-\rho_{\mathrm{ee}}$

where $a$ is the saturation parameter

$$
a=\frac{|\varepsilon|^{2}\left|\left\langle\mathrm{e}\left|Q_{\varepsilon \varepsilon}\right| \mathrm{g}\right\rangle\right|}{2 \hbar \sqrt{\Gamma_{\mathrm{e}} \Gamma_{\mathrm{eg}}}}
$$

and $\delta \tilde{E}$ is the energy detuning from the two-photon absorption resonance

$$
\delta \tilde{E}=\hbar \omega_{\mathrm{eg}}+s-2 \hbar \tilde{\omega} .
$$

1.2 POLARIZATION OF AN ATOM IN ITS REST FRAME. For the calculation of the polarization of the medium and the refractive index, we need the mean value of $D$ :

$$
\langle\mathbf{D}\rangle=\operatorname{Tr} \rho \mathbf{D}=2 \operatorname{Re} \sum_{\mathrm{j}}\left(\rho_{\mathrm{je}} \mathbf{D}_{\mathrm{ej}}+\rho_{\mathrm{jg}} \mathbf{D}_{\mathrm{gj}}\right)
$$

where use is made of $D_{\mathrm{eg}}=0$.

We obtain $\rho_{\mathrm{je}}$ and $\rho_{\mathrm{jg}}$ (as it was mentioned before) by integrating adiabatically the equations (1) giving $i \hbar \frac{\mathrm{d}}{\mathrm{d} t} \rho_{\mathrm{je}}$ and $i \hbar \frac{\mathrm{d}}{\mathrm{d} t} \rho_{\mathrm{gj}}$. The values of $\rho_{\mathrm{je}}$ and $\rho_{\mathrm{jg}}$ only depend on $\rho_{\mathrm{gg}}, \rho_{\mathrm{ge}}$ and $\rho_{\mathrm{ee}}$ which have been calculated previously (see (8)-(11)). We find for $\langle\mathbf{D}\rangle$ :

$$
\begin{aligned}
\langle\mathbf{D}\rangle=-\operatorname{Re} \varepsilon^{*} \mathrm{e}^{i \tilde{\omega} \mathrm{r}}\left[\left\langle\mathrm{e}\left|\mathbf{D} \varepsilon^{*} \frac{1}{\hbar \tilde{\omega}+E_{\mathrm{e}}-H_{0}} \mathbf{D}+\mathbf{D} \frac{1}{-\hbar \tilde{\omega}+E_{\mathrm{e}}-H_{0}} \mathbf{D} \varepsilon^{*}\right| \mathrm{e}\right\rangle \rho_{\mathrm{ee}}+\right. \\
+\left\langle\mathrm{g}\left|\mathbf{D} \varepsilon^{*} \frac{1}{\hbar \tilde{\omega}-H_{0}} \mathbf{D}+\mathbf{D} \frac{1}{-\hbar \tilde{\omega}+E_{\mathrm{e}}-H_{0}} \mathbf{D} \varepsilon^{*}\right| \mathrm{e}\right\rangle \rho_{\mathrm{eg}} \\
+\left\langle\mathrm{e}\left|\mathbf{D} \varepsilon^{*} \frac{1}{\hbar \tilde{\omega}+E_{\mathrm{e}}-H_{0}} \mathbf{D}+\mathbf{D} \frac{1}{-\hbar \tilde{\omega}-H_{0}} \mathbf{D} \varepsilon^{*}\right| \mathrm{g}\right\rangle \rho_{\mathrm{ge}} \\
\left.+\left\langle\mathrm{g}\left|\mathbf{D} \varepsilon^{*} \frac{1}{\hbar \tilde{\omega}-H_{0}} \mathbf{D}+\mathbf{D} \frac{1}{-\hbar \tilde{\omega}-H_{0}} \mathbf{D} \varepsilon^{*}\right| \mathrm{g}\right\rangle \rho_{\mathrm{gg}}\right]
\end{aligned}
$$

This expression for $\langle\mathbf{D}\rangle$ contains terms oscillating at $3 \omega$ which correspond to frequency mixing and terms oscillating at $\omega$. In the following, we only retain these last terms which must be taken into account in order to calculate the refractive index. Furthermore, we neglect in the following the antiresonant terms (those which correspond to an energy denominator equal to $\left.-\hbar \tilde{\omega}-H_{0}\right)$.

In the sections 1 and 2 we also assume that $\varepsilon$ is real and that $\mathbf{D}$ is colinear to $\varepsilon$ (it corresponds, for instance, to the case of a $\mathrm{S}-\mathrm{S}$ two-photon transition).

Introducing the notations $D=$ D. $\varepsilon$ and $Q=Q_{\varepsilon \varepsilon}$ we transform the expression (15) into :

$\langle D\rangle=-\left(Q_{\mathrm{gg}} \rho_{\mathrm{gg}}+Q_{\mathrm{ee}}^{\prime} \rho_{\mathrm{ee}}\right) E(t)-2 \operatorname{Re} \times$

$$
\times\left(\rho_{\mathrm{eg}} Q_{\mathrm{ge}} \delta^{*} \mathrm{e}^{i \tilde{\omega} t}\right) .
$$

Using $\rho_{\mathrm{gg}}=1-\rho_{\mathrm{ee}}$ and the definition (7) of the lightshift $s$, this expression can be transformed into :

$$
\begin{aligned}
\langle D\rangle=-\left(Q_{\mathrm{gg}}+\frac{4 s}{|\varepsilon|^{2}} \rho_{\mathrm{ee}}\right) & E(t)- \\
& -2 \operatorname{Re}\left(\rho_{\mathrm{eg}} Q_{\mathrm{ge}} \xi^{*} \mathrm{e}^{i \tilde{\omega} t}\right) .
\end{aligned}
$$

The first term $-Q_{\mathrm{gg}} E(t)$ corresponds to the usual linear polarization. The term $\left(4 s /|\delta|^{2}\right) \rho_{\mathrm{ee}} E(t)$ is a corrective term to the linear polarization which comes from the modification of the populations of the states $g$ and $\mathrm{e}$ due to the two-photon transition. The last expression is the term expected for two-photon dispersion because it involves the optical coherence $\rho_{\mathrm{eg}}$. Using the formulae (8)-(13), we obtain for $\langle D\rangle$ :

$$
\begin{aligned}
& \langle D\rangle=\operatorname{Re}\left(\delta \mathrm{e}^{-i \tilde{\omega} t}\right)\left(-Q_{\mathrm{gg}}+\frac{\left|Q_{\mathrm{ge}}\right|^{2}|\delta|^{2}\left(\delta \tilde{E}-s \Gamma_{\mathrm{eg}} / \Gamma_{\mathrm{e}}\right)}{2\left[\delta \tilde{E}^{2}+\hbar^{2} \Gamma_{\mathrm{eg}}^{2}\left(1+a^{2}\right)\right]}\right)+ \\
& \quad+\operatorname{Re}\left(\mathcal{E} \mathrm{e}^{-i \tilde{\omega} t} \mathrm{e}^{i \frac{\pi}{2}}\right)\left(\frac{\left|Q_{\mathrm{ge}}\right|^{2}|\delta|^{2} \hbar \Gamma_{\mathrm{eg}}}{2\left[\delta \tilde{E}^{2}+\hbar^{2} \Gamma_{\mathrm{eg}}^{2}\left(1+a^{2}\right)\right]}\right) .
\end{aligned}
$$


The first term of $\langle\mathbf{D}\rangle$ (with a phase identical to the one of the incident electric field) corresponds to the real part of the polarizability $\chi^{\prime}$ (i.e. the dispersion) while the second term corresponds to the imaginary part $\chi^{\prime \prime}$ (i.e. the absorption). This last value is the one which is classically obtained in the theory of twophoton absorption [6]. The relations between $\chi^{\prime}$ and $\chi^{\prime \prime}$ are clearly very different from the one encounted in the theory of susceptibility for a non-saturated single-photon transition. In particular the value for which $\chi^{\prime \prime}$ is maximum $(\delta \tilde{E}=0)$ does not correspond to the value for which the non-linear term of $\chi^{\prime}$ (labelled $\left.\chi_{\mathrm{NL}}^{\prime}\right)$ is equal to $0 \delta \tilde{E}=s \frac{\Gamma_{\mathrm{eg}}}{\Gamma_{\mathrm{e}}}$. Furthermore, the zero of $\chi_{\mathrm{NL}}^{\prime}$ can be very sensitive to collisional processes because generally $\Gamma_{\mathrm{eg}}$ and $\Gamma_{\mathrm{e}}$ have a different behaviour with the pressure of vapour.

Up to now, we have not taken into account the velocity distribution of the atoms. That will be done in the next sections. However, because of the possibility of cancelling the Doppler broadening in a twophoton transition [1], we shall demonstrate that most of the features found in formula (16) remain true when the velocity distribution is taken into account.

1.3 Atoms in a travelling wave. - In the case of a dilute atomic vapour interacting with a travelling wave, we have to transform the previous formulae in order to take into account the spatial dependence of the electric field and the velocities of the atoms. The electric field is now equal to :

$$
E(z, t)=\operatorname{Re} \varepsilon \exp i(k z-\omega t) .
$$

Because of the motion of the atom, $z$ depends on $t$. If we assume for the trajectory a straight path $z=V_{z} t+z_{0}$, we have to replace in the previous formulae $\widetilde{\omega}$ by $\omega-k V_{z}\left({ }^{1}\right)$. The values of $\chi^{\prime}$ and $\chi^{\prime \prime}$ are obtained by transforming the formulae into the laboratory frame and by averaging over the velocities. $N, T, m$ and $k_{\mathrm{B}}$ being respectively the number of atoms per unit volume, the temperature of the vapour, the mass of the atoms and the Boltzmann constant, we find :

$$
\begin{aligned}
& \chi^{\prime}=-\frac{N}{\varepsilon_{0}} Q_{\mathrm{gg}}+\frac{N}{\varepsilon_{0}} \int_{-\infty}^{+\infty} \mathrm{d} V_{z}\left[\frac{m}{2 \pi k_{\mathrm{B}} T}\right]^{1 / 2} \exp -\frac{m V_{z}^{2}}{2 k_{\mathrm{B}} T} \frac{\left|Q_{\mathrm{ge}}\right|^{2}\left|\varepsilon_{0}\right|^{2}\left(\delta E-s \Gamma_{\mathrm{eg}} / \Gamma_{\mathrm{e}}-2 \hbar k V_{z}\right)}{2\left[\left(\delta E-2 \hbar k V_{z}\right)^{2}+\hbar^{2} \Gamma_{\mathrm{eg}}^{2}\left(1+a^{2}\right)\right]} \\
& \chi^{\prime \prime}=\frac{N}{\varepsilon_{0}} \int_{-\infty}^{+\infty} \mathrm{d} V_{z}\left[\frac{m}{2 \pi k_{\mathrm{B}} T}\right]^{1 / 2} \exp -\frac{m V_{z}^{2}}{2 k_{\mathrm{B}} T} \frac{\left|Q_{\mathrm{ge}}\right|^{2}\left|\varepsilon_{0}\right|^{2} \hbar \Gamma_{\mathrm{eg}}}{2\left[\left(\delta E-2 \hbar k V_{z}\right)^{2}+\hbar^{2} \Gamma_{\mathrm{eg}}^{2}\left(1+a^{2}\right)\right]} .
\end{aligned}
$$

In these formulae $\delta E=\left(\hbar \omega_{\mathrm{eg}}+s-2 \hbar \omega\right)$ is the energy detuning from the two-photon resonance.

The term first in (17) corresponds to the linear susceptibility $\chi_{\mathrm{L}}$ of the vapour.

Up to now, we have not demonstrated that the plane travelling wave can be a solution of the Maxwell equations if two-photon processes are taken into account. It is easy to show that $\mathcal{E}(\mathbf{r})=\varepsilon_{0} \mathrm{e}^{i k z}$ (with $k$ independent of $z$ ) is a solution of

$$
\nabla^{2} \mathbf{E}-\frac{1}{c^{2}} \frac{\partial^{2} E}{\partial t^{2}}=\mu_{0} \frac{\partial^{2} P}{\partial t^{2}}
$$

only if $\left|\varepsilon_{0}\right|$ is constant which means that the absorption must be negligible on a range of the order of the wavelength. In that case, we can define an index

$$
n\left(\varepsilon_{0}\right)=\frac{c k}{\omega}=\left(1+\chi^{\prime}\right)^{1 / 2}
$$

which is dependent on the amplitude $\varepsilon_{0}$ of the electric field.

2. Atoms interacting with two travelling waves of opposite direction. -2.1 Polarization OF AN ATOM
INTERACTING WITH TWO WAVES OF DIFFERENT FREQUENCY. - We consider an atom which interacts in its rest frame with two electric fields whose polarizations are parallel to $\varepsilon$

$$
\begin{aligned}
& E_{1}=\operatorname{Re} \varepsilon_{1} \mathrm{e}^{-i \tilde{\omega}_{1} t} \\
& E_{2}=\operatorname{Re} \varepsilon_{2} \mathrm{e}^{-i \tilde{\omega}_{2} t} .
\end{aligned}
$$

In order to simplify the discussion, we perform several assumptions on the relative values of $\widetilde{\omega}_{1}$ and $\widetilde{\omega}_{2}$ :

(i) $\left|\tilde{\omega}_{1}-\tilde{\omega}_{2}\right| \gg \Gamma_{\text {eg }}\left(1+a^{2}\right)^{1 / 2}$ (width of the optical transition).

The condition shows that we can separate the processes corresponding to the absorption of two photons $\tilde{\omega}_{1}$, of two photons $\tilde{\omega}_{2}$ and of one photon $\tilde{\omega}_{1}$ and one photon $\tilde{\omega}_{2}$.

(ii) $\left|\widetilde{\omega}_{1}-\tilde{\omega}_{2}\right| \gg\left|\omega_{\mathrm{eg}}-\tilde{\omega}_{1}-\tilde{\omega}_{2}\right|$.

If this condition is fulfilled, it implies that the essential contribution to the non-linear polarizability has its

( $\left.{ }^{1}\right)$ Usually [1, 3], we neglect $k V_{z}$ compared to the single-photon detunings $\left|\omega_{\mathrm{jg}}-\omega\right|,\left|\omega_{\mathrm{je}}+\omega\right| \ldots$ 
origin in the quasi-resonant absorption of two different photons $\left(\tilde{\omega}_{1}\right.$ and $\left.\tilde{\omega}_{2}\right)$. This can be understood because $\omega_{\text {eg }}-2 \tilde{\omega}_{1}$ is equal to

$$
\left(\omega_{\mathrm{eg}}-\tilde{\omega}_{1}-\tilde{\omega}_{2}\right)+\left(\tilde{\omega}_{2}-\tilde{\omega}_{1}\right)
$$

which is roughly equal to $\tilde{\omega}_{2}-\tilde{\omega}_{1}$. (iii) $\left|\tilde{\omega}_{1}-\tilde{\omega}_{2}\right| \ll\left|\omega_{\text {jg }}-\tilde{\omega}_{1}\right|,\left|\omega_{\text {jg }}-\tilde{\omega}_{2}\right|$.

If this condition is fulfilled, we can use the same two-photon operators $Q$ and $Q^{\prime}$ (defined for an average value of $\omega \simeq \frac{\widetilde{\omega}_{1}+\tilde{\omega}_{2}}{2}$.

If those three assumptions are valid, we can as in section 1.1 derive an effective hamiltonian :

$$
H_{\mathrm{eff}}=\left(\begin{array}{ll}
E_{\mathrm{e}}+\frac{1}{4} Q_{\mathrm{e}}^{\prime}\left(\left|\varepsilon_{1}\right|^{2}+\left|\varepsilon_{2}\right|^{2}\right) & \frac{1}{2} Q_{\mathrm{eg}} \varepsilon_{1} \varepsilon_{2} \exp -i\left(\tilde{\omega}_{1}+\tilde{\omega}_{2}\right) t \\
\frac{1}{2} Q_{\mathrm{ge}} \varepsilon_{1}^{*} \varepsilon_{2}^{*} \exp i\left(\tilde{\omega}_{1}+\tilde{\omega}_{2}\right) t & \frac{1}{4} Q_{\mathrm{gg}}\left(\left|\varepsilon_{1}\right|^{2}+\left|\varepsilon_{2}\right|^{2}\right)
\end{array}\right) .
$$

Performing the same calculations as in section 1, we obtain the mean value of the electric dipole moment $\langle D\rangle$. We separate in $\langle D\rangle$ the components oscillating at $\tilde{\omega}_{1}\left(\langle D\rangle_{\tilde{\omega}_{1}}\right)$ and $\tilde{\omega}_{2}\left(\langle D\rangle_{\tilde{\omega}_{2}}\right)$. For instance, we obtain for $\langle D\rangle_{\tilde{\omega}_{1}}$ :

$$
\begin{array}{r}
\langle D\rangle_{\tilde{\omega}_{1}}=\left[\operatorname{Re}\left(\mathcal{E}_{1} \mathrm{e}^{-i \tilde{\omega}_{1} t}\right)\left(-Q_{\mathrm{gg}}+\frac{\left|Q_{\mathrm{ge}}\right|^{2}\left|\mathcal{E}_{2}\right|^{2}\left(\delta \tilde{E}-2 s_{1} \Gamma_{\mathrm{eg}} / \Gamma_{\mathrm{e}}\right)}{(\delta \tilde{E})^{2}+\Gamma_{\mathrm{eg}}^{2}\left(1+a_{12}^{2}\right)}\right)+\right. \\
\left.+\operatorname{Re}\left(\varepsilon_{1} \mathrm{e}^{-i \tilde{\omega}_{1} t} \mathrm{e}^{i \frac{\pi}{2}}\right) \frac{\left|Q_{\mathrm{ge}}\right|^{2}\left|\varepsilon_{2}\right|^{2} \hbar \Gamma_{\mathrm{eg}}}{(\delta \tilde{E})^{2}+\Gamma_{\mathrm{eg}}^{2}\left(1+a_{12}^{2}\right)}\right]
\end{array}
$$

with

$$
\begin{gathered}
\delta \tilde{E}=\hbar\left(\tilde{\omega}_{\mathrm{eg}}-\tilde{\omega}_{1}-\tilde{\omega}_{2}\right)+s_{1}+s_{2} \\
s_{1}=\frac{1}{4}\left(Q_{\mathrm{ee}}^{\prime}-Q_{\mathrm{gg}}\right)\left|\varepsilon_{1}\right|^{2} \\
s_{2}=\frac{1}{4}\left(Q_{\mathrm{ee}}^{\prime}-Q_{\mathrm{gg}}\right)\left|\delta_{2}\right|^{2} \\
a_{12}=\frac{\left|Q_{\mathrm{eg}}\right|\left|\varepsilon_{1} \varepsilon_{2}\right|}{\hbar \sqrt{\Gamma_{\mathrm{e}} \Gamma_{\mathrm{eg}}}} .
\end{gathered}
$$

There are two minor differences between this value of $\langle D\rangle_{\tilde{\omega}_{1}}$ and the one obtained in a travelling wave (formula (16)). The non-linear dependence of $\langle D\rangle$ which was proportional to $\left|Q_{\mathrm{ge}}\right|^{2} / 2$ is now proportional to $\left|Q_{\mathrm{ge}}\right|^{2}$. Moreover the correction of the dispersion due to the transfer of population $\left(s \Gamma_{\mathrm{eg}} / \Gamma_{\mathrm{e}}\right)$ is also multiplied by 2 . These two factors 2 have the same physical origin. They come from the fact that it is now possible for the atom to reach the excited state by two different ways [3] : absorbing first $\tilde{\omega}_{1}$ and then $\tilde{\omega}_{2}$ or absorbing the photons in the opposite order. At those two ways it corresponds a factor 2 on the coherence effects and a factor 4 on the population effects.

2.2 ATOMS IN TWO TRAVELLING WAVES OF OPPOSITE DIRECTION. - We now consider moving atoms interacting with two travelling waves propagating in opposite directions :

$$
\begin{aligned}
& \mathbf{E}_{1}=\varepsilon E_{1} \cos \left(k_{1} z-\omega_{1} t\right) \\
& \mathbf{E}_{2}=\varepsilon E_{2} \cos \left(k_{2} z+\omega_{2} t\right) .
\end{aligned}
$$

We assume that the conditions (i), (ii), (iii) of paragraph 2.1 are valid for each atom in its rest frame. We can then find as in paragraph 1.3 a complex susceptibility. We have two essential terms in this susceptibility, the first corresponds to the Fourier component at $\omega_{1}$, the second to the one at $\omega_{2}$. For instance, we obtain for $\chi\left(\omega_{1}\right)=\chi_{1}=\chi_{1}^{\prime}+i \chi_{1}^{\prime \prime}$ the following results :

$$
\begin{aligned}
& \chi_{1}^{\prime}=-\frac{N}{\varepsilon_{0}} Q_{\mathrm{gg}}+\frac{N}{\varepsilon_{0}} \int_{-\infty}^{+\infty} \mathrm{d} V_{z}\left[\frac{m}{2 \pi k_{\mathrm{B}} T}\right]^{1 / 2} \exp -\frac{m V_{z}^{2}}{2 k_{\mathrm{B}} T} \frac{\left|Q_{\mathrm{ge}}\right|^{2}\left|\varepsilon_{2}\right|^{2}\left(\delta E-2 s_{1} \Gamma_{\mathrm{eg}} / \Gamma_{\mathrm{e}}+\hbar\left(k_{1}-k_{2}\right) V_{z}\right)}{\left(\delta E+\hbar\left(k_{1}-k_{2}\right) V_{z}\right)^{2}+\hbar^{2} \Gamma_{\mathrm{eg}}^{2}\left(1+a_{12}^{2}\right)} \\
& \chi_{1}^{\prime \prime}=\frac{N}{\varepsilon_{0}} \int_{-\infty}^{+\infty} \mathrm{d} V_{z}\left[\frac{m}{2 \pi k_{\mathrm{B}} T}\right]^{1 / 2} \exp -\frac{m V_{z}^{2}}{2 k_{\mathrm{B}} T} \frac{\left|Q_{\mathrm{ge}}\right|^{2}\left|\varepsilon_{2}\right|^{2} \hbar \Gamma_{\mathrm{eg}}}{\left(\delta E+\hbar\left(k_{1}-k_{2}\right) V_{z}\right)^{2}+\hbar^{2} \Gamma_{\mathrm{eg}}^{2}\left(1+a_{12}^{2}\right)}
\end{aligned}
$$

$\delta E$ being equal to $\hbar\left(\omega_{\mathrm{eg}}-\omega_{1}-\omega_{2}\right)+s_{1}+s_{2}$.

These expressions are similar to the ones obtained by Liao and Bjorklund [2] but take into account terms which are of higher order in electric field. As in this previous work we obtained a susceptibility at the 
pulsation $\omega_{1}$ which depends on the magnitude of the other field $\varepsilon_{2}$.

It can also be noticed that we have the following relation : $\chi_{1}^{\prime \prime} \varepsilon_{1}^{2}=\chi_{2}^{\prime \prime} \varepsilon_{2}^{2}$ which corresponds to the fact that we have to absorb the same number of photons of each wave in order to excite the atoms.

As in section 1.3, we can show that the plane waves $\varepsilon_{1} \exp \left(k_{1} z-\omega_{1} t\right)$ and $\varepsilon_{2} \exp i\left(k_{2} z+\omega_{2} t\right)$ are solutions of the Maxwell equations if the absorption can be neglected. We then find a refraction index $n_{1}$ which is at the lowest order in the electric field only function of the intensity $I_{2}$ of the opposite wave [2].

2.3 ATOMS IN A STANDING WAVE : SMALL ENERGY DETUNING. - We now consider the case where the two oppositely travelling waves have the same amplitude and the same frequency in the laboratory frame. It corresponds to the case of a standing wave. If the energy detuning $\hbar\left(\omega_{\mathrm{eg}}-2 \omega\right)$ is small compared to the Doppler width of the two-photon transition, we can apply the results of the previous section with no essential changes.

If the Doppler width $k u$ is large compared to the energy detuning $\left|\omega_{\mathrm{eg}}-2 \omega\right|$, it means that for most of the atoms, in their rest frame, the assumptions of paragraph 2.1 are satisfied. The pulsations in this frame $\tilde{\omega}_{1}$ and $\tilde{\omega}_{2}$ are respectively equal to $\omega-k V_{z}$ and $\omega+k V_{z}$ and $\left|\tilde{\omega}_{1}-\tilde{\omega}_{2}\right|=\left|2 k V_{z}\right|$ is generally larger than $\left|\omega_{\text {eg }}-\tilde{\omega}_{1}-\tilde{\omega}_{2}\right|$.

If the Doppler width is larger than the natural width of the g-e transition (even with saturation), the first assumption is also satisfied for most of the atoms. The number of velocity groups for which the hypothesis are not satisfied being very small, we can use the results of paragraph 2.1 with the further simplification that $\omega_{1}=\omega_{2}=\omega$. We obtained in the case of two opposite waves of same amplitude $\left(\varepsilon_{1}=\varepsilon_{2}=\varepsilon\right)$ :

$$
\chi^{\prime}=-\frac{N}{\varepsilon_{0}} Q_{\mathrm{gg}}+\frac{N}{\varepsilon_{0}} \frac{\left|Q_{\mathrm{ge}}\right|^{2}|\delta|^{2}\left(\delta E-2 s\left(\Gamma_{\mathrm{eg}} / \Gamma_{\mathrm{e}}\right)\right)}{(\delta E)^{2}+\hbar^{2} \Gamma_{\mathrm{eg}}^{2}\left(1+a^{2}\right)}
$$$$
\chi^{\prime \prime}=\frac{N}{\varepsilon_{0}} \frac{\left|Q_{\mathrm{ge}}\right|^{2}|\delta|^{2} \hbar \Gamma_{\mathrm{eg}}}{(\delta E)^{2}+\hbar^{2} \Gamma_{\mathrm{eg}}^{2}\left(1+a^{2}\right)}
$$

with

$$
\begin{gathered}
\delta E=\hbar\left(\omega_{\mathrm{eg}}-2 \omega\right)+2 s \\
a=\frac{\left|Q_{\mathrm{eg}}\right||\mathcal{E}|^{2}}{\hbar \sqrt{\Gamma_{\mathrm{e}} \Gamma_{\mathrm{eg}}}} .
\end{gathered}
$$

The first comment that we can perform on these formulae is that we can obtain a very large value of $\chi^{\prime}$ because the cancellation of the Doppler broadening permits to obtain the same response for all the atoms. In particular for a small value of $\delta E\left(\sim \hbar \Gamma_{\mathrm{eg}}\right)$ the nonlinear part of the susceptibility is about $k \bar{V} / \Gamma_{\mathrm{eg}}$ ( $k \bar{V}$ being the Doppler width) larger than the value calculated in a travelling wave.
It is also possible to observe here that the relations between $\chi^{\prime}$ and $\chi^{\prime \prime}$ are different from those obtained in the single-photon case. If $\chi^{\prime \prime}$ keeps a lorentzian shape, its maximum being at

$$
\omega_{\max }=\left(\frac{\omega_{\mathrm{eg}}}{2}+\frac{s}{\hbar}\right),
$$

$\chi^{\prime}$ has not the usual shape of a dispersion curve (see Fig. 1), in particular it must be noticed that the value for which $\chi_{\mathrm{NL}}^{\prime}=0$ differs from $\omega_{\max }$. As noticed previously the behaviour of $\chi^{\prime}$ comes from the fact that we take into account in our calculations the modification of the linear susceptibility due to the variation of population of the ground and excited levels.
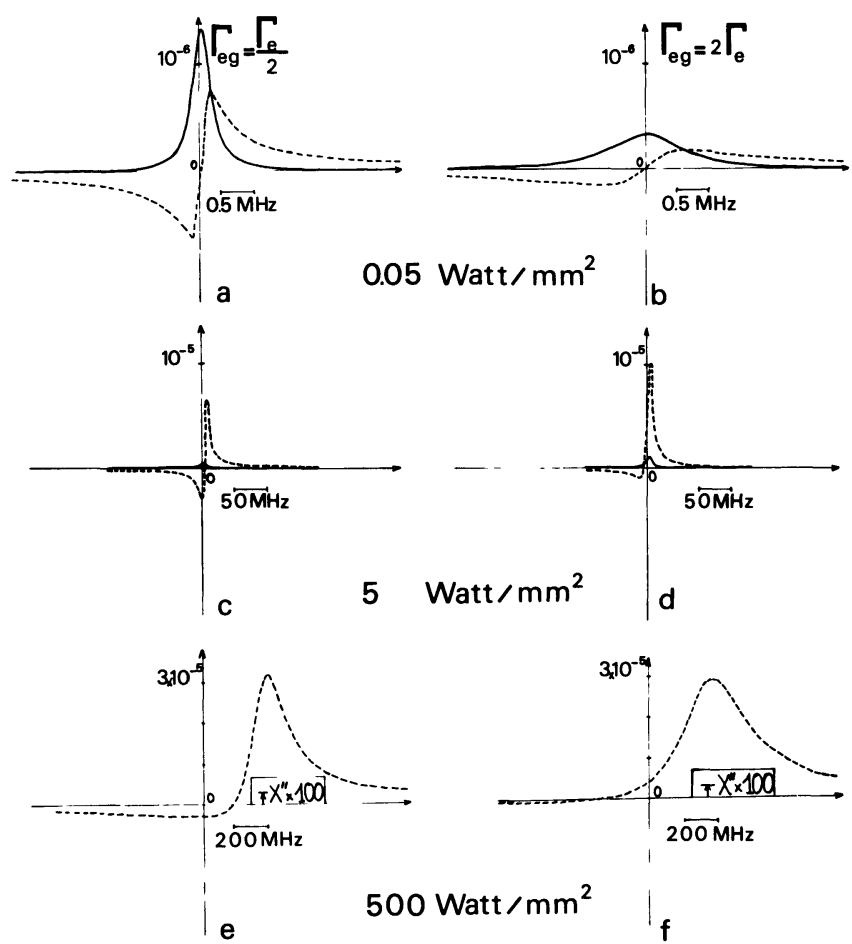

Fig. 1. - Value of the non-linear susceptibilities $\chi^{\prime \prime}$ (full line) and $\chi^{\prime}$ (dashed lines) as a function of the frequency detuning

$$
\left(\omega_{\mathrm{eg}}-2 \omega\right) / 2 \pi
$$

in the case of the 5S-5D two-photon transition in $\mathrm{Rb}$ for $T=200^{\circ} \mathrm{C}$. The curves a, c and e correspond to a relaxation rate for the optical coherence $\Gamma_{\mathrm{eg}}$ equal to $\Gamma_{\mathrm{e}} / 2$. The curves b, d and f to $\Gamma_{\mathrm{eg}}=2 \Gamma_{\mathrm{e}}$. The curves $\mathrm{a}$ and $\mathrm{b}$ correspond to an incident power $I$ equal to $0.05 \mathrm{~W} / \mathrm{mm}^{2}$, the curves $\mathrm{c}$ and $\mathrm{d}$ to $I=5 \mathrm{~W} / \mathrm{mm}^{2}$, the curves e and $\mathrm{f}$ to $I=5 \times 10^{2} \mathrm{~W} / \mathrm{mm}^{2}$. In this last case, the value of $\chi^{\prime \prime}$ is too small to be shown on the same scale as $\chi^{\prime}$. It can be observed that for large value of $I$ and of $\Gamma_{\mathrm{eg}} / \Gamma_{\mathrm{e}}, \chi^{\prime}$ keeps a sign practically constant in the region where its value is important.

If the energy detuning is small compared to the Doppler width and if the absorption is negligible on a range of the order of a wavelength, the same argument as above shows that the standing wave $2 \varepsilon \cos k z \cos \omega t$ is a solution of the Maxwell equations and we obtain a refractive index equal to $\sqrt{1+\chi^{\prime}} \simeq 1+\frac{\chi}{2}$ in the case of a vapour. 
2.4 ATOMS IN A STANDING WAVE : LARGE ENERGY DETUNING. - In the case of a large detuning, the condition (ii) of paragraph 2.1 is no more valid. It means that all the energy detunings $\hbar\left(\omega_{\mathrm{eg}}-\tilde{\omega}_{1}-\tilde{\omega}_{2}\right)$, $\hbar\left(\omega_{\mathrm{eg}}-2 \tilde{\omega}_{1}\right), \hbar\left(\omega_{\mathrm{eg}}-2 \tilde{\omega}_{2}\right)$ are now of the same order of magnitude. It is now difficult to find an exact solution because we have to take these different processes into account simultaneously. Moreover, we show hereafter that the standing wave (in that situation) is generally not a solution of the Maxwell equations. More precisely the spatial dependence of the polarization is different from the one of the standing wave and it is not possible to define a refractive index. We can interpret the difference with the previous situation in the following way : the Rabi period (which is close to $\left.\left(\omega_{\mathrm{eg}}-2 \omega\right)^{-1}\right)$ is very small compared to the time of flight of the atom between two nodes of the standing wave (time $\sim(k \bar{V})^{-1}$ ). Because the polarization arises from a non-linear process, it follows that the spatial dependence of the polarization is different from the one of the standing wave. Such an effect does not occur if the assumptions of the previous section (2.3) are valid; in this case, the time of flight $(k \bar{V})^{-1}$ is short compared to the Rabi period and the motion of the atom averages the intensity variations of the standing wave [6]. As we have seen above, it results in the possibility to obtain an index for the standing wave.

In the case of an energy detuning large compared to the Doppler width, it is not possible to obtain an exact solution for the Maxwell equations because the susceptibility $\chi$ now depends on the position. However, as it is shown in Appendix, for a dilute vapour contained in a cell of dimension $L$ we obtain a quantity $\tilde{n}$ which has the same behaviour as the refractive index if $\left|\tilde{n}-n_{0}\right| L / \hat{\lambda} \ll 1\left(n_{0}\right.$ is the usual refractive index and $\lambda$ is the wavelength). If the field is assumed to be the sum of two oppositely propagating waves of amplitude $\varepsilon_{1}$ and $\varepsilon_{2}$, the value of $\tilde{n}_{1}$ is equal to (see Appendix) :

$\tilde{n}_{1}=n_{0}+\frac{N\left|Q_{\mathrm{ge}}\right|^{2}}{2 \varepsilon_{0} \hbar\left(\omega_{\mathrm{eg}}-2 \omega\right)} \times\left(\frac{\left|\varepsilon_{1}\right|^{2}}{2}+\left|\varepsilon_{2}\right|^{2}\right)$.
We can notice that at this lowest order in electric field, the effective index looks as the sum of the contribution to the index of the travelling wave 1 alone and the contribution to the index of wave 1 perturbed by wave 2 . It means that we simply add the effect of dispersion due to the detunings $\hbar\left(\omega_{\mathrm{eg}}-2 \tilde{\omega}_{1}\right)$ and $\hbar\left(\omega_{\text {eg }}-\tilde{\omega}_{1}-\tilde{\omega}_{2}\right)$. In this last case the factor 2 has its usual origin from the two possible paths for absorbing the different photons $\left(\tilde{\omega}_{1}\right)$ and $\left(\tilde{\omega}_{2}\right)$.

\section{Case of different polarizations for the two beams.} - In the previous section, we have discussed the problem of the interaction of a standing wave with an atom near a two-photon transition. It was possible to attain an exact solution with a non-perturbational treatment because of the two important assumptions :

(i) we have considered two non-degenerate levels,

(ii) we have considered the same polarization for the two oppositely propagating travelling waves.

However, there are many situations where these assumptions are not valid. For these cases, we present below a perturbational treatment and we obtain the refractive index at the lowest order in electric field. We then describe two different types of anisotropy which can be induced by one beam on another beam of different polarization.

3.1 THEORY OF THE SUSCEPTIBILITY. - We consider an atom with a ground level $\mathrm{g}$ of angular momentum $J_{\mathbf{g}}$ (the sublevels are labelled according to their magnetic quantum numbers $m_{\mathrm{g}}$ ). The excited state e has an angular momentum $J_{\mathrm{e}}$. In its rest frame the atom interacts with two waves propagating in opposite directions

$$
\begin{aligned}
& \mathbf{E}_{1}=\operatorname{Re} \varepsilon_{1} \varepsilon_{1} \mathrm{e}^{-i \tilde{\omega}_{1} t} \\
& \mathbf{E}_{2}=\operatorname{Re} \varepsilon_{2} \varepsilon_{2} \mathrm{e}^{-i \tilde{\omega}_{2} t}
\end{aligned}
$$

where $\varepsilon_{1}$ and $\varepsilon_{2}$ can be complex polarizations. We assume for the present section the same hypothesis as in paragraph 2.1 .

In order to find the component of the electric dipole moment oscillating at frequency $\widetilde{\omega}_{2}$, we perform a calculation identical to the one already done in paragraph 1.2 and we obtain :

$$
\begin{array}{r}
\left\langle D\left(\tilde{\omega}_{2}\right)\right\rangle=-\operatorname{Re} \sum_{m_{\mathbf{g}}, m_{\mathrm{e}}}\left\langle m_{\mathrm{e}}|\rho| m_{\mathrm{g}}\right\rangle \boldsymbol{\varepsilon}_{1}^{*} \mathrm{e}^{i \tilde{\omega}_{1} t}\left[\left\langle m_{\mathrm{g}}\left|\mathbf{D} \frac{1}{\hbar \omega-H_{0}} \mathbf{D} \boldsymbol{\varepsilon}_{1}^{*}+\mathbf{D} \varepsilon_{1}^{*} \frac{1}{\hbar \omega-H_{0}} \mathbf{D}\right| m_{\mathrm{e}}\right\rangle\right]- \\
-\operatorname{Re} \sum_{m_{\mathrm{g}}} \frac{\varepsilon_{2} \mathrm{e}^{-i \tilde{\omega}_{2 t}}}{2 J_{\mathrm{g}}+1}\left\langle m_{\mathrm{g}}\left|\mathbf{D} \frac{1}{\hbar \omega-H_{0}} \mathbf{D} \varepsilon_{2}\right| m_{\mathrm{g}}\right\rangle .
\end{array}
$$

The second term of this expression corresponds to the usual linear susceptibility. In the following we focus our attention in the first term : the non-linear part of $\left\langle D\left(\tilde{\omega}_{2}\right)\right\rangle$ labelled $\left\langle D_{\mathrm{NL}}\left(\tilde{\omega}_{2}\right)\right\rangle$. By evaluating $\left\langle m_{\mathrm{e}}|\rho| m_{\mathrm{g}}\right\rangle$ 
at the lowest order of perturbation (the ground level is assumed to be not polarized), we obtain :

$$
\begin{array}{r}
\left\langle D_{\mathrm{NL}}\left(\tilde{\omega}_{2}\right)\right\rangle=\operatorname{Re} \frac{\left|\varepsilon_{1}\right|^{2} \varepsilon_{2} \mathrm{e}^{-i \widetilde{\omega}_{2} t}}{\hbar\left(\omega_{\mathrm{eg}}-2 \omega-i \Gamma_{\mathrm{eg}}\right)} \cdot \frac{1}{2 J_{\mathrm{g}}+1} \sum_{m_{\mathrm{g}}, m_{\mathrm{e}}}\left[\left\langle m_{\mathrm{g}}\left|Q_{\varepsilon_{1}^{*} \varepsilon_{2}^{*}}^{\mathrm{S}}\right| m_{\mathrm{e}}\right\rangle\left\langle m_{\mathrm{e}}\left|Q_{\varepsilon_{1} \varepsilon_{2}}^{\mathrm{s}}\right| m_{\mathrm{g}}\right\rangle \varepsilon_{2}+\right. \\
\left.+\left\langle m_{\mathrm{g}}\left|Q_{\varepsilon_{1}^{*} \varepsilon_{2}^{*}}^{\mathrm{S}}\right| m_{\mathrm{e}}\right\rangle\left\langle m_{\mathrm{e}}\left|Q_{\varepsilon_{1} \varepsilon_{2}}^{\mathrm{s}}\right| m_{\mathrm{g}}\right\rangle \varepsilon_{2}^{\prime}\right] .
\end{array}
$$

We have introduced in this formula the symmetrical two-photon operator [5]

$$
Q_{\varepsilon_{1} \varepsilon_{2}}^{\mathrm{S}}=\frac{1}{2}\left[Q_{\varepsilon_{1} \varepsilon_{2}}+Q_{\varepsilon_{2} \varepsilon_{1}}\right] \text {. }
$$

$\varepsilon_{2}^{\prime}$ is the polarization orthogonal to $\varepsilon_{2}$ (which means that $\varepsilon_{2}^{\prime} \cdot \varepsilon_{2}^{*}=0$ ) in the plane orthogonal to $\mathbf{e}_{z}$ (direction of propagation of the waves). In theory, we should introduce a component of $\left\langle\mathbf{D}_{\mathrm{NL}}\left(\boldsymbol{\omega}_{2}\right)\right\rangle$ along $\mathbf{e}_{z}$. However the application of the formulae demonstrated below for the component along $\varepsilon_{2}^{\prime}$ shows that the component along $\mathrm{O} z$ is equal to 0 . So we don't keep it in the formula. The circular frequency $\omega$ is equal to $\left(\tilde{\omega}_{1}+\tilde{\omega}_{2}\right) / 2$. Apart from the anisotropic terms, the difference between (24) and the formula obtained previously in the non-perturbational theory $(\$ 2.1)$ comes from the fact that all the effects related to the light-shift and the saturation are now neglected.

We can obtain a further simplification in the expression of $\left\langle\widetilde{D}_{\mathrm{NL}}\left(\widetilde{\omega}_{2}\right)\right\rangle$ by introducing the standard components of the two-photon operator [5, 7] (see formula (13) of [5])

$$
{ }^{\mathrm{eg}} Q_{\varepsilon_{1} \varepsilon_{2}}^{\mathrm{s}}=P_{\mathrm{e}} Q_{\varepsilon_{1} \varepsilon_{2}}^{\mathrm{s}} P_{\mathrm{g}}=\sum_{k=0,2} \sum_{q} a_{q}^{k}\left(\varepsilon_{1}, \varepsilon_{2}\right) \frac{\left\langle\mathrm{e} J_{\mathrm{e}}\left\|Q^{k}\right\| g J_{\mathrm{g}}\right\rangle}{\sqrt{2 k+1}} T_{q}^{\mathrm{k}}\left(\mathrm{e} J_{\mathrm{e}}, \mathrm{g} J_{\mathrm{g}}\right)
$$

where $P_{\mathrm{e}}$ and $P_{\mathrm{g}}$ are the projectors on the e $J_{\mathrm{e}}$ and $\mathrm{g} J_{\mathrm{g}}$ subspaces. The coefficients $a_{q}^{k}\left(\varepsilon_{1}, \varepsilon_{2}\right)$ are presented in [5].

Reporting this expression of ${ }^{\mathrm{eg}} Q_{\varepsilon_{1} \varepsilon_{2}}^{\mathrm{S}}$ in formula (24) and using the orthogonality relation of the ClebschGordan coefficients, we find (this demonstration is very similar to the one presented in the case of absorption in $[5], \S 1.3 .1)$

$$
\begin{aligned}
\left\langle D_{\mathrm{NL}}\left(\tilde{\omega}_{2}\right)\right\rangle=\operatorname{Re} & \frac{\left|\varepsilon_{1}\right|^{2} \varepsilon_{2} \mathrm{e}^{-i \omega_{2} t}}{\hbar\left(\omega_{\mathrm{eg}}-2 \omega-i \Gamma_{\mathrm{eg}}\right)} \times \frac{1}{2 J_{\mathrm{g}}+1} \times \\
& \times \sum_{k=0,2} \frac{\left|\left\langle\mathrm{e} J_{\mathrm{e}}\left\|Q^{k}\right\| \mathrm{g} J_{\mathrm{g}}\right\rangle\right|^{2}}{2 k+1}\left[\varepsilon_{2} \sum_{q}\left|a_{q}^{k}\left(\varepsilon_{1}, \varepsilon_{2}\right)\right|^{2}+\varepsilon_{2}^{\prime} \sum_{q} a_{q}^{k}\left(\varepsilon_{1}, \varepsilon_{2}\right) a_{q}^{k *}\left(\varepsilon_{1}, \boldsymbol{\varepsilon}_{2}^{\prime}\right)\right] .
\end{aligned}
$$

It is possible to obtain an explicit value for the summation over $q$. Some values have already been reported [5], the other ones can be calculated easily using a theory identical to the one developed in [8]. We find (the coefficients $a_{q}^{1}$ are equal to $\left.0[5]\right)$ :

$$
\begin{aligned}
\left|a_{0}^{0}\left(\varepsilon_{1}, \varepsilon_{2}\right)\right|^{2} & =\frac{1}{3}\left|\varepsilon_{1} \cdot \varepsilon_{2}\right|^{2} \\
\sum_{k=0,2} \sum_{q}\left|a_{q}^{k}\left(\varepsilon_{1}, \varepsilon_{2}\right)\right|^{2} & =\frac{1}{2}+\frac{1}{2}\left|\varepsilon_{1} \cdot \varepsilon_{2}^{*}\right|^{2} \\
a_{0}^{0}\left(\varepsilon_{1}, \varepsilon_{2}\right) a_{0}^{0 *}\left(\varepsilon_{1}, \varepsilon_{2}^{\prime}\right) & =\frac{1}{3}\left(\varepsilon_{1} \cdot \varepsilon_{2}\right)\left(\varepsilon_{1} \cdot \varepsilon_{2}^{\prime}\right)^{*} \\
\sum_{k=0,2} \sum_{q} a_{q}^{k}\left(\varepsilon_{1}, \varepsilon_{2}\right) a_{q}^{k *}\left(\varepsilon_{1}, \varepsilon_{2}^{\prime}\right) & =\frac{1}{2}\left(\varepsilon_{1} \cdot \varepsilon_{2}^{\prime *}\right)\left(\varepsilon_{1}^{*} \cdot \varepsilon_{2}\right) .
\end{aligned}
$$

(In order to demonstrate this last formula, we have used $\varepsilon_{2} \cdot \varepsilon_{2}^{\prime *}=0$ ). Thus the formulae (25) and (26) permit to calculate the angular dependence of the non-linear susceptibility without any difficulty $\left({ }^{2}\right)$.

For the sake of simplicity, we assume now that the intensity of one beam is much larger than the intensity of the other (for instance $I_{1} \gg I_{2}$ ). In that case, the polarization $\varepsilon_{1}$ of the beam 1 is only very slightly modified during its propagation and we can take it as a constant.

It appears obviously on formula (25) that the dipole moment $\left\langle D_{\mathrm{NL}}\left(\tilde{\omega}_{2}\right)\right\rangle$ varies linearly with the electric field $\varepsilon_{2}$. Thus we can define a refractive index for the wave $\varepsilon_{2}$. We can also (because of this linearity) add two solutions $\boldsymbol{E}_{2}$ and $\boldsymbol{E}_{2}^{\prime}$ (provided that $I_{2}$ and $I_{2}^{\prime} \ddot{<} \ddot{<} I_{1}$ ) to find another solution.

Our problem consists in finding the eigenvalue of the tensor of susceptibility (see formula (25)). We now solve this problem for two important particular cases : the case where the intense beam $\varepsilon_{1}$ is circular and the case where it is linear.

$\left({ }^{2}\right)$ In order to obtain the susceptibility $\chi$, we must come back to the laboratory frame and then average on the velocity distribution (see section 1.4). We assume in this section that the two oppositely travelling waves have wave vectors of the same order of magnitude such that the residual Doppler effect $\left|k_{1}-k_{2}\right| \bar{V}$ is much smaller than $\Gamma_{\mathrm{eg}}$ which permits to simplify the average over the velocities. 
3.2 Polarization Rotation INDUCED By a CIRCUlarly POlarized beAm. - We assume that the intense beam 1 is circularly polarized $\left(\varepsilon_{1}=\mathbf{e}_{+}\right.$for instance). By applying formulae (25) and (26), it appears immediately that the eigenstates of the tensor of susceptibility correspond to the two opposite circular polarizations. More precisely, if we assume that $\left|\omega_{\mathrm{eg}}-2 \omega\right| \gg \Gamma_{\mathrm{eg}}$, we find the following values for the refractive index $n_{+}$and $n_{-}$ corresponding to a polarization $\boldsymbol{\varepsilon}_{2}$ identical to $\mathbf{e}_{+}$or orthogonal to it $\left(\mathbf{e}_{-}\right)$

$$
\begin{aligned}
& n_{+}=n_{0}-\frac{N}{2 \varepsilon_{0}} \cdot \frac{I_{1}}{\hbar\left(\omega_{\mathrm{eg}}-2 \omega\right)} \cdot \frac{1}{\left(2 J_{\mathrm{g}}+1\right)} \cdot \frac{\left|\left\langle\mathrm{e} J_{\mathrm{e}}\left\|Q^{2}\right\| \mathrm{g} J_{\mathrm{g}}\right\rangle\right|^{2}}{5} \\
& n_{-}=n_{0}-\frac{N}{2 \varepsilon_{0}} \frac{I_{1}}{\hbar\left(\omega_{\mathrm{eg}}-2 \omega\right)} \frac{1}{\left(2 J_{\mathrm{g}}+1\right)}\left[\frac{1}{6} \frac{\left|\left\langle\mathrm{e} J_{\mathrm{e}}\left\|Q^{2}\right\| \mathrm{g} J_{\mathrm{g}}\right\rangle\right|^{2}}{5}+\frac{1}{3}\left|\left\langle\mathrm{e} J_{\mathrm{e}}\left\|Q^{0}\right\| \mathrm{g} J_{\mathrm{g}}\right\rangle\right|^{2}\right]
\end{aligned}
$$

$n_{0}$ being the ordinary index at this wavelength.

It appears obviously that the refractive index corresponding to the two opposite circular polarizations $\mathbf{e}_{+}$ and $\mathbf{e}_{-}$are practically always different. It follows that if the beam (2) has a linear polarization at the entrance of the cell, this linear polarization rotates during its propagation in a way identical to the case of the Faraday effect. This effect has been observed by Liao and Bjorklund [2] in the case of the $3 \mathrm{~S}_{1 / 2}-5 \mathrm{~S}_{1 / 2}$ transition in sodium. It must be noticed that the effect is not restricted to the case of a S-S transition (even if it is the most obvious case because $\left\langle\mathrm{e} L_{\mathrm{e}}\left\|Q^{2}\right\| \mathrm{g} L_{\mathrm{g}}\right\rangle=0$ and that $n_{+}=n_{0}$ ). This effect occurs for almost all the transitions. In particular if $J_{\mathrm{g}} \neq J_{\mathrm{e}}$ only the tensor of rank $k=2$ couple the ground and the excited states and it is easy to see that the nonlinear part of the refractive index is then 6 times larger for $n_{+}$than for $n_{-}$.

Addition of hyperfine coupling can be dealt with in the same manner as in reference [5].

3.3 BIREFRINGENCE INDUCED BY A LINEARLY POLARIZED BEAM. - We now assume that the intense beam 1 is linearly polarized $\left(\varepsilon_{1}=\mathbf{e}_{x}\right.$ for instance). By applying formulae (25) and (26), we find that the eigenstates of the tensor of susceptibility correspond to the polarizations $\mathbf{e}_{x}$ and $\mathbf{e}_{y}$. We find

$$
\begin{aligned}
& n_{x}=n_{0}-\frac{N}{2 \varepsilon_{0}} \cdot \frac{I_{1}}{\hbar\left(\omega_{\mathrm{eg}}-2 \omega\right)} \frac{1}{\left(2 J_{\mathrm{g}}+1\right)}\left[\frac{2}{3} \frac{\left|\left\langle\mathrm{e} J_{\mathrm{e}}\left\|Q^{2}\right\| \mathrm{g} J_{\mathrm{g}}\right\rangle\right|^{2}}{5}+\frac{1}{3}\left|\left\langle\mathrm{e} J_{\mathrm{e}}\left\|Q^{0}\right\| \mathrm{g} J_{\mathrm{g}}\right\rangle\right|^{2}\right] \\
& n_{y}=n_{0}-\frac{N}{2 \varepsilon_{0}} \cdot \frac{I_{1}}{\hbar\left(\omega_{\mathrm{eg}}-2 \omega\right)} \cdot \frac{1}{2 J_{\mathrm{g}}+1} \cdot \frac{1}{2} \frac{\left|\left\langle\mathrm{e} J_{\mathrm{e}}\left\|Q^{2}\right\| \mathrm{g} J_{\mathrm{g}}\right\rangle\right|^{2}}{5} .
\end{aligned}
$$

The refractive index for waves polarized parallel to the axes $\mathrm{O} x$ and $\mathrm{O} y$ are practically always different. We thus obtain a birefringent medium.

4. Bistability near a doppler-free two-photon transition. - The dependence of the index of refraction with the intensity may also lead to the observation of bistability [10]. The possibility of observing bistability near a two-photon resonance has already been suggested in a nice paper of Arecchi and Politi [11]. However, even if these authors were aware of the interest of the standing wave pattern in order to eliminate the Doppler broadening, their theory is performed for the case of a travelling wave in a ring cavity. Furthermore, they essentially consider the case of twophoton absorption bistability. We feel that twophoton dispersive bistability is easier to observe experimentally. For instance, if the light-shift $s$ is different from 0 , as it is usually the case, it is not possible to find a situation where the absorptive part appears alone (see formulae (21) and (22)). On the other hand, there are many experimental conditions (see Fig. 1) where $\chi^{\prime \prime}$ is closed to 0 while $\chi^{\prime}$ has an important value.

Many Doppler-free two-photon experiments are performed inside a Pérot-Fabry cavity [9]. Such a system close to a two-photon resonance is potentially bistable (which means that for the same input power in the cavity, one may observe different outputs according to the history of the system). Up to now, bistability has not been observed in these conditions because the density of the atoms (or the power of the laser) was not sufficient. But, as we shall demonstrate it hereafter, a bistable behaviour can occur for several two-photon transitions in alkalis using commercial c.w. dye laser.

By comparison with previous experiments on bistability performed with an atomic vapour [10], the Doppler-free two-photon experiments present several advantages :

- because the energy detuning from the two-photon transition is the same for all the atoms [12], all the atoms $\left({ }^{3}\right)$ will have the same behaviour. They will contribute either to the dispersion or to the absorption in the same way;

- the formulae for the refractive index are more

$\left({ }^{3}\right)$ We neglect the small fraction of atoms which can absorb two photons of the same progressive wave. 
simple in the Doppler-free two-photon case because the average on the Doppler distribution can be obviously calculated. The atomic system, and in particular its response, is thus easier to understand and we can get more information on the bistable device itself.

In order to precise in which experimental conditions bistability can be experimentally observed, we perform hereafter a calculation of the order of magnitude of the dephasage $\delta \Phi=n_{2} \frac{\omega}{c} l$ (where $n_{2}$ is the non-linear part of the refractive index and $l$ is the length of the cell). At the lowest order in electric field $n_{2}$ is equal to (see formula (28)) :

$$
n_{2}=\frac{N}{2 \varepsilon_{0}} \cdot I \times b
$$

with

$$
\begin{aligned}
b=\frac{1}{\hbar\left(\omega_{\mathrm{eg}}-2 \omega\right)} \cdot & \frac{1}{\left(2 J_{\mathrm{g}}+1\right)} \cdot \\
& \times\left[\frac{2}{3} \frac{\left|\left\langle\mathrm{e} J_{\mathrm{e}}\left\|Q^{2}\right\| \mathrm{g} J_{\mathrm{g}}\right\rangle\right|^{2}}{5}+\frac{1}{3}\right. \\
& \left.\times\left|\left\langle\mathrm{e} J_{\mathrm{e}}\left\|Q^{0}\right\| \mathrm{g} J_{\mathrm{g}}\right\rangle\right|^{2}\right] .
\end{aligned}
$$

In many experimental situations, it is more convenient to use a Pérot-Fabry with spherical mirrors instead of a Pérot-Fabry with plane mirrors. In the case of a gaussian beam, the intensity is not uniform and the index of refraction $n_{2}$ is now depending on $z$. For the present discussion we do not consider the problem of self focussing and we just calculate the value of the dephasage $\delta \Phi$ along the axis of symmetry of the gaussian beam :

$$
\delta \Phi=\int_{-1 / 2}^{1 / 2} \frac{\omega}{c} n_{2}(z) \mathrm{d} z
$$

To calculate $\delta \Phi$ we use the value (29) of $n_{2}$ with

$$
I=\frac{4}{\varepsilon_{0} c \pi} \frac{P}{W^{2}(z)}
$$

where $P$ is the power of the laser and $W(z)$ is the radius of the gaussian beam (more precisely it is the distance from the axis where the electric field is divided by $e$ )

$$
W(z)^{2}=W_{0}^{2}\left(1+\frac{z^{2}}{z_{\mathrm{R}}^{2}}\right) .
$$

The waist $W_{0}$ is the smallest value of $W$ and $z_{\mathrm{R}}=\pi W_{0}^{2} / \lambda$. We find for $\delta \Phi$ :

$$
\delta \Phi=4 \frac{N}{\varepsilon_{0}^{2} c^{2}} \omega \frac{P}{\lambda} a \operatorname{Arctg} \frac{1}{2 z_{\mathrm{R}}} .
$$

We observe that if $l \gtrsim z_{\mathrm{R}}, \delta \Phi$ has practically reached its asymptotic value :

$$
\delta \Phi_{\mathrm{as}}=\frac{N}{\varepsilon_{0}^{2} c^{3}} \omega^{2} P b
$$

From an experimental point of view it appears that there are only two factors which can be modified : the power $P$ and the density $N$ of atoms. If one increases the focussing, the value of $\delta \Phi$ will not increase very much if the higher terms in power of the intensity (see formula (21)) can be neglected.

In order to observe a bistable behaviour we must attain a value of $\delta \Phi$ of the order of $2 \pi / F$ where $F$ is the finesse of the Pérot-Fabry ( $P$ being the power inside the cavity).

A rough calculation performed in the case of the $5 \mathrm{~S}_{1 / 2}-5 \mathrm{D}_{5 / 2}$ two-photon transition in $\mathrm{Rb}(\hat{\lambda}=7779 \AA)$ shows that those conditions can be attained with $F \sim 20$, an incident power of $0.1 \mathrm{~W}$ and a pressure of rubidium of the order of $10^{-2}$ torr, the frequency detuning from the resonance being equal to $30 \mathrm{MHz}$. These conditions can be attained easily and we expect to obtain soon an experimental confirmation of the theory.

Acknowledgments. - We are very indebted to Dr. E. Giacobino for the helpful and stimulating discussions we have together and for the precious advices about the manuscript.

Appendix. - In the case of an energy detuning large compared to the Doppler width, it is possible to perform the calculations by making the assumption that the atom looks still [6]. The reason for that is that the atom undergoes a lot of Rabi precessions between two nodes of the electromagnetic field. In that case, we can apply formula (16) but with an electric field $\varepsilon$ which is now dependent on $z$. It results in a susceptibility $\chi(z)$ which is dependent on $z$. More precisely, we split $\chi(z)$ into two terms, the linear susceptibility $\chi_{\mathrm{L}}$ which does not depend on $z$ and the non-linear term $\chi_{\mathrm{NL}}(z)=\chi(z)-\chi_{\mathrm{L}}$.

We now try to find an approximate solution for the Maxwell equation for the electric field :

$$
\frac{\mathrm{d}^{2} \varepsilon}{\mathrm{d} z^{2}}+\frac{\omega^{2}}{c^{2}} \varepsilon=-\frac{\omega^{2}}{c^{2}} \chi(z) \varepsilon
$$

This equation can be written as :

$$
\frac{\mathrm{d}^{2} \mathcal{E}}{\mathrm{d} z^{2}}+k_{0}^{2} \delta=-k_{0}^{2} \chi(z) \varepsilon
$$

with :

$$
k_{0}^{2}=\frac{\omega^{2}}{c^{2}}
$$

Using the Green function

$$
G_{+}(z)=\frac{1}{2 i k} \exp (i k-\varepsilon)|z|
$$


we find :

$$
\mathcal{E}(z)=\varepsilon_{0}(z)+\frac{i k_{0}}{2} \int_{-\infty}^{+\infty} \mathrm{d} z^{\prime} \mathrm{e}^{\left(i k_{0}-\varepsilon\right)\left|z-z^{\prime}\right|} \chi_{\mathrm{NL}}\left(z^{\prime}\right) \mathcal{E}\left(z^{\prime}\right)
$$

where $\varepsilon_{0}(z)$ is the unperturbed electric field (which means the field without any atoms). Its value is equal to :

$$
\mathcal{E}_{0}(z)=\left|\mathcal{E}_{1}\right| \mathrm{e}^{i \varphi_{1}} \mathrm{e}^{i k_{0} z}+\left|\mathcal{E}_{2}\right| \mathrm{e}^{i \varphi_{2}} \mathrm{e}^{-i k_{0} z} .
$$

If the exact solution is close to $\varepsilon_{0}(z)$, we obtain an approximate value of the correction using the Born approximation which consists in replacing in (A.1) $\chi\left(z^{\prime}\right) \mathcal{E}\left(z^{\prime}\right)$ by its unperturbed value $\chi^{0}\left(z^{\prime}\right) \varepsilon_{0}\left(z^{\prime}\right)\left({ }^{4}\right)$.

We assume that the vapour is contained inside a cell limited by the planes $z=0$ and $z=L$. The field outside the cell is equal, at the Born approximation, to :

$$
\begin{aligned}
& z<0: \mathcal{E}^{\mathrm{B}}(z)=\varepsilon_{0}(z)+\frac{i k_{0}}{2} \mathrm{e}^{-i k_{0} z} \int_{0}^{\mathrm{L}} \mathrm{d} z^{\prime} \chi^{0}\left(z^{\prime}\right)\left[\left|\mathcal{E}_{1}\right| \mathrm{e}^{i \varphi_{1}} \mathrm{e}^{2 i k_{0} z^{\prime}}+\left|\mathcal{E}_{2}\right| \mathrm{e}^{i \varphi_{2}}\right] \\
& z>L: \mathcal{E}^{\mathrm{B}}(z)=\varepsilon_{0}(z)+\frac{i k_{0}}{2} \mathrm{e}^{i k_{0} z} \int_{0}^{L} \mathrm{~d} z^{\prime} \chi^{0}\left(z^{\prime}\right)\left[\left|\varepsilon_{1}\right| \mathrm{e}^{i \varphi_{1}}+\left|\delta_{2}\right| \mathrm{e}^{i \varphi_{2}} \mathrm{e}^{-2 i k_{0} z^{\prime}}\right] .
\end{aligned}
$$

Because of the dependence of $\chi^{0}\left(z^{\prime}\right)$ on $\left|\varepsilon_{0}\left(z^{\prime}\right)\right|^{2}$ it can be easily shown that most of the integrated terms oscillate with $L$ while only one term grows monotonically with $L$. This last term is preponderant if the dimension of the cell $L$ is much larger than $1 / k_{0}$ (which is of the order of the wavelength). This condition is usually fulfilled in our experiments in the optical domain. We define :

$$
\begin{aligned}
\delta k & =\frac{k_{0}}{2} \lim _{L \rightarrow+\infty} \frac{1}{L} \int_{0}^{L} \mathrm{~d} z \chi^{0}\left(z^{\prime}\right) \\
\delta k^{\prime} & =\frac{k_{0}}{2} \lim _{L \rightarrow+\infty} \frac{1}{L} \int_{0}^{L} \mathrm{~d} z^{\prime} \chi^{0}\left(z^{\prime}\right) \exp 2 i k_{0} z^{\prime} .
\end{aligned}
$$

At this approximation, the electric field, outside the cell, takes the simple value (we assume that there is no absorption) :

$$
\begin{aligned}
& z<0 \quad \mathcal{E}^{\mathrm{B}}(z)=\left|\mathcal{E}_{1}\right| \mathrm{e}^{i \varphi_{1}} \mathrm{e}^{i k_{0} z}+\left|\mathcal{E}_{2}\right| \mathrm{e}^{i \varphi_{2}} \mathrm{e}^{-i k_{0} z}\left[1+i \delta k L+i \frac{\left|\mathcal{E}_{1}\right| \mathrm{e}^{i \varphi_{1}}}{\left|\delta_{2}\right| \mathrm{e}^{i \varphi_{2}}} \delta k^{\prime} L\right] \\
& z<L \quad \mathcal{E}^{\mathrm{B}}(z)=\left|\delta_{1}\right| \mathrm{e}^{i \varphi_{1}} \mathrm{e}^{i k_{0} z}\left[1+i \delta k L+i \frac{\left|\mathcal{E}_{2}\right| \mathrm{e}^{i \varphi_{2}}}{\left|\delta_{1}\right| \mathrm{e}^{i \varphi_{1}}} \delta k^{\prime *} L\right]+\left|\delta_{2}\right| \mathrm{e}^{i \varphi_{2}} \mathrm{e}^{-i k_{0} z}
\end{aligned}
$$

As we neglect absorption, it is easy to verify that $\delta k$ and $\delta k^{\prime} \mathrm{e}^{i\left(\varphi_{1}-\varphi_{2}\right)}$ are real quantities (it is a consequence of the dependence of $\chi\left(z^{\prime}\right)$ on $\left.\left|\delta\left(z^{\prime}\right)\right|^{2}\right)$. If $\delta k L$ and $\left|\frac{\varepsilon_{1}}{\varepsilon_{2}}\right| \delta k^{\prime} L$ are small compared to 1 , we can interpret the quantity $(1+i x)$ (with $x \gtrless<1)$ as the beginning of the expansion of $\mathrm{e}^{i x}$. By this method, we can define two pseudo-refractive index $\tilde{n}_{1}$ and $\tilde{n}_{2}$, one for each wave propagating in the medium :

$$
\begin{aligned}
& \tilde{n}_{1}=1+\frac{1}{k_{0}}\left(\delta k+\frac{\left|\delta_{2}\right| \mathrm{e}^{i \varphi_{2}}}{\left|\delta_{1}\right| \mathrm{e}^{i \varphi_{1}}} \delta k^{\prime *}\right) \\
& \tilde{n}_{2}=1+\frac{1}{k_{0}}\left(\delta k+\frac{\left|\delta_{1}\right| \mathrm{e}^{i \varphi_{1}}}{\left|\delta_{2}\right| \mathrm{e}^{i \varphi_{2}}} \delta k^{\prime}\right) .
\end{aligned}
$$

Those pseudo-refractive index can be used to calcu- late the apparent optical length of the medium as long as the previous conditions are fulfilled. Using the relations (16), (A.4), (A.5), we obtain at the lowest order in the electric field :

$$
\begin{aligned}
& \tilde{n}_{1}=1-\frac{N Q_{\mathrm{gg}}}{2 \varepsilon_{0}}+\frac{N\left|Q_{\mathrm{ge}}\right|^{2}}{2 \varepsilon_{0} \hbar\left(\omega_{\mathrm{eg}}-\right.}2 \omega) \\
& \times\left[\frac{\left|\varepsilon_{1}\right|^{2}}{2}+\left|\varepsilon_{2}\right|^{2}\right]
\end{aligned}
$$

and a symmetric expression for $\tilde{n}_{2}$.

$\left({ }^{4}\right)$ As the non-linear susceptibility $\chi\left(z^{\prime}\right)$ depends on $z^{\prime}$ through the value of the electric field $\&\left(z^{\prime}\right)$, the notation $\chi^{0}\left(z^{\prime}\right)$ means that we perform the calculation of $\chi\left[\varepsilon\left(z^{\prime}\right)\right]$ with the unperturbed value of the electric field : $\chi^{0}\left(z^{\prime}\right)=\chi\left[\varepsilon_{0}\left(z^{\prime}\right)\right]$. 


\section{References}

[1] Grynberg, G. and Cagnac, B., Rep. Prog. Phys. 40 (1977) 791.

[2] Liao, P. F. and Bjorklund, G. C., Phys. Rev. A 15 (1977) 2009.

[3] Cagnac, B., Grynberg, G. and Biraben, F., J. Physique 34 (1973) 845.

[4] Harvey, K. C. and StoḯchefF, B. P., Phys. Rev. Lett. 38 (1977) 537.

[5] Grynberg, G., Biraben, F., Giacobino, E. et Cagnac, B., J. Physique 38 (1977) 629.

[6] Grynderg, G., J. Physique 40 (1979) 657.
[7] Grynberg, G., Thèse, Paris (1976) (CNRS AO 12497).

[8] Grynberg, G., J. Physique 40 (1979) 965.

[9] Giacobino, E., Biraben, F., Grynberg, G. et Cagnac, B., J. Physique 38 (1977) 623.

[10] Gibis, H. M., Mc Call, S. L. and Venkatesan, I. N. C. Phys. Rev. Lett. 36 (1976) 1135.

[11] ARecCHI, F. T. and Politi, A., Lett. Nuovo Cimento 23 (1978) 65.

[12] Bassini, M., Biraben, F., Cagnac, B. and Grynberg, G., Opt. Commun. 21 (1977). 